\title{
Serum calcium level is related to both intima-media thickness and carotid atherosclerosis: a neglect risk factor in obese/overweight subjects
}

\author{
Tiziana Montalcini*, Gaetano Gorgone and Arturo Pujia
}

\begin{abstract}
Background: Experimental studies suggested that high serum calcium may be important in the pathogenesis of vascular diseases. Since calcium seems to affect specifically the cerebrovascular district, aim of this study was to determine the relation between serum calcium levels, within normal range, and subclinical atherosclerosis in the carotid arteries, in a population of obese/overweight subjects.

Methods: In our retrospective study we included 472 subjects (59\% female) with body mass index equal to or more than $25 \mathrm{~kg} / \mathrm{m}^{2}$. They underwent a physical examination, a biochemical assessment (including calcium evaluation) and a B-mode ultrasonography of the extracranial carotid arteries to detect carotid atherosclerosis presence and to measure intima-media thickness.

Results: Mean age of the population was $50 \pm 12$ years. Prevalence of the Carotid atherosclerosis was $40 \%$. Mean carotid intima-media thickness was $0,66 \pm 0,18 \mathrm{~mm}$. The univariate and multivariate analysis showed an association between calcium and carotid intima-media thickness $(p=0,035)$. We divided the population in serum calcium tertiles. We found an higher carotid atherosclerosis prevalence in the III tertile in comparison to that of the I tertile $(p=0,039)$.

Conclusions: In this study we found a positive relation between serum calcium levels, within normal range, and subclinical atherosclerosis in the carotid arteries, in a population of obese/overweight subjects. It is important to consider the impact of the serum calcium levels in the overall risk assessment of patients, at least in obese subjects.
\end{abstract}

Keywords: Calcium, Atherosclerosis, Carotid, Obesity, Ultrasound

\section{Background}

Experimental studies suggested that calcium-phosphate metabolism could influence the pathogenesis of vascular diseases. In fact, several studies showed an increased cardiovascular morbidity and mortality in primary hyperparathyroidism (PHPT)[1-8]. Furthermore, increased carotid intima-media thickness (CIMT), a subclinical predictor of coronary and cerebrovascular events [9-12], was found in patients with PHPT and high calcium levels [13], suggesting a causative role of hypercalcemia. However, in most

\footnotetext{
* Correspondence: tmontalcini@unicz.it

Clinical Nutrition Unit, Department of Medical and Surgical Science, School of Medicine, University Magna Grecia, Viale S Venuta, 88100, Catanzaro, Italy
}

patients with PHPT, serum calcium levels is in normal range [9]. A study showed that serum calcium level, within the normal range, was positively associated to carotid atherosclerotic plaques presence [14]. Consequently, to date, the link between the serum calcium level and the cardiovascular diseases has not been fully elucidated. Furthermore, calcium and phosphate levels seem to exert differential effects, depending on the type and location of the vascular bed [15]. In particular it was demonstrated that calcium seems to affect specifically the cerebrovascular district [15]. For this reason our objective was to investigate on the possible relation between serum calcium levels, within normal range, and the presence of carotid atherosclerosis. Furthermore, since a recent research [16] 
proposing the participation of the calcium-sensing receptor (CaSR) as a possible link between obesity and inflammation, had attracted our attention, we chose to investigate the relation between calcium and carotid atherosclerosis in a population of overweight/obese subjects.

\section{Methods}

In our retrospective study participants were recruited from subjects underwent an health-screening tests in our hospital clinic for the presence of one or more cardiovascular risk factors (obesity, hyperlipidemia, hypertension, diabetes, smoking).

A total of 640 individuals, with at least one cardiovascular risk factor, underwent an ultrasonography of the carotid arteries during a period from the year 2008 to 2011. We excluded 168 normal weight individuals (40\% males), therefore in this study 472 subjects (59\% women; $41 \%$ men) were included. All individuals were Caucasian and underwent a collection of the medical history, by a standardized questionnaire administered to obtain information about current and past medication use, smoking habits and presence of cardiovascular disease. They underwent, also, a physical examination including the evaluation of the body mass index (BMI)(calculated as weight, in $\mathrm{kg}$, divided by square of height, in meter); the measurement of the waist circumferences (WC)(a measuring tape was used for WC, measured right below the ribs); the measurement of the systemic blood pressure in both arms by a sphygmomanometer (systolic and diastolic blood pressure - SBP and DBP). The ankle-brachial systolic pressure index (Winsor Index) was determined by dividing the highest of the posterior tibial or dorsalis pedis systolic blood pressure by the highest brachial pressure. On the basis of the clinical history, no subjects had symptomatic cardiovascular disease.

Venous blood was collected after fasting overnight into vacutainer tubes (Becton \& Dickinson) and centrifuged within $4 \mathrm{~h}$. Serum glucose, creatinine, total cholesterol, high density lipoprotein cholesterol, triglycerides and calcium were measured with enzymatic colorimetric test (see Additional file 1: Supplemental materials). Serum Calcium were measured by Schwarzenbach test (see Additional file 1: Supplemental materials). Quality control was assessed daily for all determinations. The investigation conforms to the principles outlined in the Declaration of Helsinki. All patients provided informed consent to participate in our research and to use their data.

The following criteria were used to define the cardiovascular risk factors; diabetes: fasting blood glucose $\geq 126 \mathrm{mg} / \mathrm{dl}$ or antidiabetic treatment; hyperlipidemia: total cholesterol $>200 \mathrm{mg} / \mathrm{dl}$ and/or triglycerides $>200 \mathrm{mg} / \mathrm{dl}$ or lipid lowering drugs use; hypertension: systolic blood pressure $\geq 140 \mathrm{mmHg}$ and/or diastolic blood pressure $\geq 90 \mathrm{mmHg}$ or antihypertensive treatment; smoking: present smokers; overweight: $\mathrm{BMI} \geq 25$ $<30 \mathrm{Kg} / \mathrm{m}^{2}$, obesity: $\mathrm{BMI} \geq 30 \mathrm{~kg} / \mathrm{m}^{2}$.

\section{Ultrasound}

The subjects underwent a B-mode ultrasonography of the extracranial carotid arteries by use of a duplex system (an high resolution ultrasound instrument "Advanced Technology Laboratories” -ATL, High Definition Imaging -HDI 5000 with a 5- to $12-\mathrm{MHz}$ linear array multifrequency transducer). All the examinations were performed by the same ultrasonographer blinded to clinical information. All patients rested in the supine position for at least $10 \mathrm{~min}$ before the study and were kept in this position during the procedure. Electrocardiographic (ECG) leads were attached to the ultrasound recorder for on-line continuous heart rate monitoring. The right and left common (CCA) and internal carotid arteries (including bifurcations) were evaluated with the head of the subjects turned away from the sonographer and the neck extended with mild rotation. The CIMT, defined as the distance between the intimal-luminal interface and the medial-adventitial interface, was measured as previously described [17]. Briefly, in posterior approach and with the sound beam set perpendicular to the arterial surface, $1 \mathrm{~cm}$ from the bifurcation, three longitudinal measurements of IMT were completed on the right and left common carotid arteries far-wall, at sites free of any discrete plaques. The mean of the three right and left longitudinal measurements was then calculated. Then, we calculated and used for statistical analysis the mean CIMT between right and left CCA. Plaque, detected in longitudinal and transverse planes with anterior, lateral and posterior approaches, was defined as an echogenic focal structure encroaching into the vessel lumen of at least $50 \%$ of the surrounding CIMT value. Stenosis was defined as a peak systolic velocity $>120 \mathrm{~cm} / \mathrm{s}$ and occlusion was defined as absence of Doppler signal. According to these criteria, subjects were considered as normal if no lesion was detected, or having carotid atherosclerosis when a plaque, stenosis or occlusion was detected in at least one segment of common, bifurcation or internal carotid artery. The coefficient variation of the methods was $3.3 \%$.

\section{Statistical analysis}

Data are reported as mean \pm S.D. The $\chi 2$-test was used to compare the prevalence among the groups. Univariate analysis was performed to identify the factors related to CIMT. The Multivariate regression analysis was performed to test for confounding variables and data were adjusted for gender. The Logistic regression analysis was used to test variables correlated to carotid atherosclerosis presence, including only the factors different at 
comparison ( $t$-test) between subjects with and without carotid atherosclerosis. Furthermore, the $X^{2}$-test was performed to compare the prevalence of the carotid atherosclerosis between "calcium tertiles" of population (between I and III tertile). Significant differences were assumed to be present at $p<0.05$. All comparisons were performed using the SPSS 17.0 for Windows $233 \mathrm{~S}$. Wacker Drive, Chicago, Illinois 60606, USA.

\section{Results}

The mean age of the population was $50 \pm 12$. The BMI was $33 \pm 6$. The characteristics of the overall population are showed in Table 1. Subjects treated with lipid lowering drugs were $28.6 \%$. Table 2 shows the Multivariate regression analysis, adjusted for gender, including the variables correlated to CIMT at univariate analysis (age, glucose, SBP, DBP calcium, WC - data no showed). This analysis confirmed the association between calcium and CIMT, as well as, age, PAS, WC with CIMT (Table 2). In this population the prevalence of the Atherosclerotic was $40 \%$ (Table 1). After $t$-test between subjects with and without carotid atherosclerosis (Table 3), we performed a Logistic regression analysis with Carotid Atherosclerosis as dependent variable (Table 4). In this analysis, in the first two models performed, including age and gender (I model), and age, gender and WC (II

Table 1 Characteristics of the population

\begin{tabular}{|c|c|c|}
\hline Variable & Mean & SD \\
\hline Age (years) & 50 & 12 \\
\hline BMI & 33,3 & 5,9 \\
\hline Calcium (mg/dl) & 9,4 & 0,4 \\
\hline Creatinine(mg/dl) & 0,8 & 0,6 \\
\hline Glucose(mg/dl) & 101 & 26 \\
\hline TCholesterol(mg/dl) & 220 & 44 \\
\hline LDLcholesterol(mg/dl) & 141 & 56 \\
\hline $\mathrm{HDL}(\mathrm{mg} / \mathrm{dl})$ & 50 & 14 \\
\hline Trygliceride(mg/dl) & 174,46 & 106,9 \\
\hline CIMTmean (mm) & 0,66 & 0,18 \\
\hline IWright & 1,06 & 0,006 \\
\hline IWleft & 1,07 & 0,007 \\
\hline Waist Circum(cm) & 104,28 & 13,30 \\
\hline $\mathrm{DBP}(\mathrm{mmHg})$ & 77,05 & 9,21 \\
\hline $\mathrm{SBP}(\mathrm{mmHg})$ & 125,19 & 14,65 \\
\hline Carotid atherosclerosis (\%) & 40 & \\
\hline diabetes(\%) & 9,7 & \\
\hline Hyperlipidemia(\%) & 47 & \\
\hline hypertension(\%) & 25,8 & \\
\hline smokers(\%) & 7,4 & \\
\hline Female (\%) & 59 & \\
\hline
\end{tabular}

Table 2 Multivariate regression analysis

\begin{tabular}{lcccc}
\hline Dependent Variable: CIMT & SE & Beta & $\mathbf{t}$ & $\mathbf{p}$ \\
\hline Age & 0,001 & 0,432 & 10,16 & $<0,001$ \\
SBP & 0,001 & 0191 & 4,50 & $<0,001$ \\
WC & 0,001 & 0,101 & 2,53 & 0,012 \\
calcium & 0,019 & 0,084 & 2,11 & 0,035 \\
\hline
\end{tabular}

(the data are adjusted for gender)

Excluded variables: PAD, glucose (factors correlated to CIMT at univariate analysis-data no shown).

model) calcium was correlated to Carotid Atherosclerosis. However, when (in the third model) glucose and SBP were included in the analysis, calcium was excluded (data no showed). Finally, we divided the population in serum calcium tertiles. Figure 1 depicts the higher carotid atherosclerosis prevalence of the III tertile in comparison to that of the I tertile $(\mathrm{p}=0,039)$.

\section{Discussion}

In this study we found a positive relation between serum calcium levels, within normal range, and CIMT. As expected,CIMT was, also, associated, to the classical risk factors like age, gender, glucose, SBP, DBP and WC. However, after adjustment for confounding factors in the multivariate analysis, the association between calcium and CIMT remained, as well as, for age, SBP and WC only (Table 2). Furthermore, in this study we showed an higher carotid atherosclerosis prevalence in the population group having high serum calcium level (III tertile)in comparison to group with low serum calcium level (I tertile) [Figure 1].

This study may have a relevant place among the studies already present in the literature since we evaluated a large number of subjects (472 individuals), with a $\mathrm{BMI}>25$ (overweight/obese) excluding subjects having pathological values of serum calcium. Furthermore, our study is important since investigated, at the same time, the relation between calcium and the various manifestations of the

Table 3 Comparison (t-test)between subjects with and without carotid atherosclerosis

\begin{tabular}{lccc}
\hline Variables & $\begin{array}{c}\text { Atherosclerosis } \\
\text { presence }\end{array}$ & $\begin{array}{c}\text { Atherosclerosis } \\
\text { absence }\end{array}$ & $\mathbf{p}$ \\
\hline AGE (years) & $57,42 \pm 8$ & $45 \pm 11$ & $<0,001$ \\
SBP(mmHg) & $129 \pm 15$ & $122 \pm 14$ & $<0,001$ \\
DBP(mmHg) & $77 \pm 10$ & $77 \pm 9$ & 0,334 \\
WC (cm) & $103 \pm 13$ & $106 \pm 14$ & 0,049 \\
BMl & $32 \pm 5$ & $34 \pm 6$ & $<0,001$ \\
Calcium (mg/dl) & $9,4 \pm 0,3$ & $9,3 \pm 0,4$ & 0,029 \\
Tot cholesterol (mg/dl) & $222 \pm 46$ & $217 \pm 43$ & 0,116 \\
HDL (mg/dl) & $51 \pm 14$ & $50 \pm 13$ & 0,413 \\
\hline
\end{tabular}


Table 4 Logistic regression analysis

\begin{tabular}{lcccccc}
\hline \multicolumn{7}{c}{ Dependent variable: Carotid Atherosclerosis } \\
\hline Variable & B & S.E. & Wald & Sig & R & Exp(B) \\
AGE & 0,15 & 0,01 & 95,23 & $<0,001$ & 0,38 & 1,16 \\
CALCIUM & 0,63 & 0,30 & 4,34 & 0,03 & 0,06 & 1,89 \\
GENDER & 1,19 & 0,26 & 21,00 & $<0,001$ & 0,17 & 3,29 \\
& & \multicolumn{7}{c}{ II model } \\
AGE & 0,15 & 0,01 & 95,50 & $<0,001$ & 0,38 & 1,16 \\
CALCIUM & 0,58 & 0,30 & 3,56 & 0,05 & 0,05 & 1,79 \\
GENDER & 1,28 & 0,26 & 23,11 & $<0,001$ & 0,18 & 3,60 \\
WC & $-0,02$ & 0,00 & 7,78 & $<0,001$ & $-0,09$ & 0,97 \\
\hline
\end{tabular}

atherosclerotic process in the carotid district (i.e.: CIMT and plaques).

Similarly to other studies $[12,15,18]$ the parathyroid hormone (PTH) measurement was not available. However, we had the objective to investigate on the possible association of serum calcium and subclinical atherosclerosis at carotid site, with others classical risk factors and not other factors.

Several investigations demonstrated the association between serum calcium and stroke, but not with coronary heart disease, in contrast to phosphate [15]. Although the cause of this divergence is uncertain, it was speculate that calcium-phosphate levels could exert differential effects, depending on the type and location of the vascular bed [15]. Thus, our finding is in line with the concept that the effects of the serum calcium are prominent for the cerebral district than for other sites [15].

The majority of cross-sectional studies indicated that elevated levels of established cardiovascular risk factors are associated with an increased CIMT [19-21] including obesity and the insulin resistance condition. Certainly, our study was not designed to explain the mechanisms

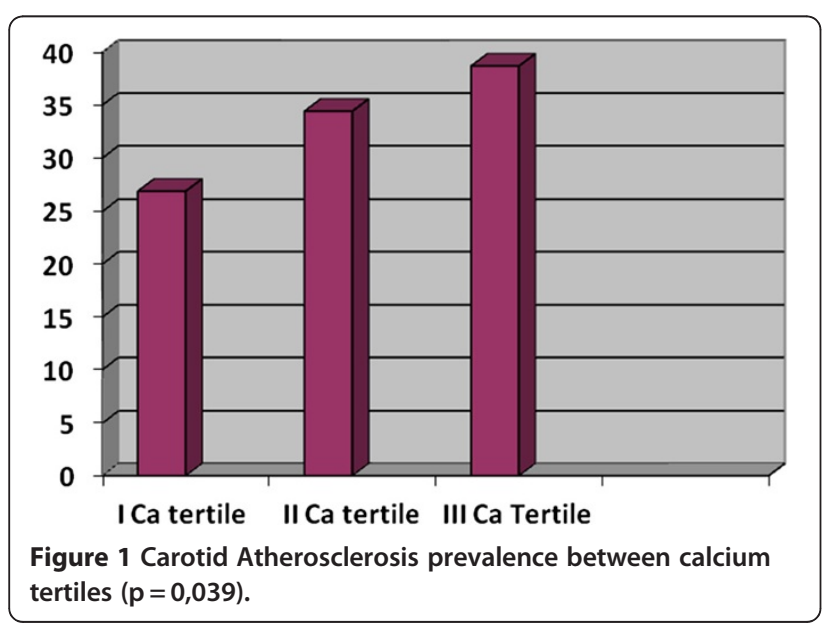

underlying the association finding in this study, however, some hypothesis may be advanced.

Several observations showed that serum calcium level, in the normal range, affect the risk of having Metabolic Syndrome (MetS) and diabetes [22]. This association may suggests a first possible mechanism by which calcium affect the intima-media thickness in our study, confirmed also by our finding of the strong relation between WC, a factor linked to MetS, and CIMT. Moreover, in our study there was a positive relation between serum calcium and glucose $(\mathrm{p}=0,022 ; \mathrm{r}=0,166$ - data no showed).

Furthermore, recently, Cifuentes et al. [16] reported that obesity-associated proinflammatory cytokines increased the CaSR protein expression in primary human adipocytes as well as in LS14 human adipose cell line. CaSR is a seven transmembrane receptor which plays a central role in regulating calcium homeostasis. In particular the activation of the CaSR maintains serum calcium at normal levels [23]. CaSR is a multifunctional protein inhibiting basal lipolysis in adipocytes [23], regulating the smooth muscle cells of vessels [24], involved also in the ischemic neuronal death [25]. All these observations could support the link between serum calcium, within normal range, and the CIMT. Furthermore, it was showed that obesity, as well as, the metabolic syndrome condition are associated to high level of serum fetuin-A, a protein playing an important role in bone metabolism, metabolic disorders, and central nervous system disorders such as ischemic stroke and neurodegenerative diseases [26,27]. Actually it is unknown whether fetuin-A is an exacerbating or a protective factor in the cardiovascular system, however, it may represent the link between inflammation, calcium and atherosclerosis in obesity, needing further investigations.

Our study has several strengths and limitations. The study population was relatively large and the young population studied should be useful for the early identification of the subclinical carotid artery disease. Our study investigated, also, the various manifestations of the atherosclerotic process in the carotid district.

A limitation of our study, similarly to other studies $[12,18]$, may be that PTH and vitamin D levels were not available, as well as, fetuin A measurement.

\section{Conclusions}

We believe it is important to consider the impact of the serum calcium levels in the overall risk assessment of patients, at least in obese individuals. Despite a number of studies on this issue, actually the blood calcium level is considered a neglect risk factor for vasculature, thus, we think is important to perform other studies on this factor. It is unclear whether this finding will have a therapeutic implication. For now, it could clarify some morphological changes of specific vascular districts. 


\section{Additional file}

\section{Additional file 1: Supplemental materials.}

\section{Abbreviation}

PHPT: Primary hyperparathyroidism; CIMT: Carotid intima-media thickness; CaSR: Calcium-sensing receptor; BMI: Body mass index; WC: Waist circumferences; SBP: Systolic blood pressure; DBP: Diastolic blood pressure.

\section{Competing interest}

There is no conflict of interest that could be perceived as prejudicing the impartiality of the research reported.

\section{Authors' contributions}

All authors participated sufficiently in the work to take public responsibility for its content. All authors read and approved the final manuscript.

\section{Funding}

This research did not receive any specific grant from any funding agency in the public, commercial or not-for-profit sector.

Received: 29 March 2012 Accepted: 23 May 2012

Published: 6 June 2012

\section{References}

1. Palmér M, Adami HO, Bergström R, Akerström $G$, Ljunghall S: Mortality after surgery for primary hyperparathyroidism: a follow-up of 441 patients operated on from 1956 to 1979. Surgery 1987, 102:1-7.

2. Hedback G, Oden A, Tisell LE: The influence of surgery on the risk of death in patients with primary hyperparathyroidism. World J Surg 1991, 15:399-405.

3. Hedback G, Tisell LE, Bengtsson BA, Hedman I, Oden A: Premature death in patients operated on for primary hyperparathyroidism. World J Surg 1990, 14:829-835.

4. Hedbäck G, Oden A: Survival of patients operated on for primary hyperparathyroidism. Surgery 1999, 125:240-241.

5. Hedbäck G, Oden A: Increased risk of death from primary hyperparathyroidism: an update. Eur J Clin Invest 1998, 28:271-276.

6. Nilsson IL, Yin L, Lundgren E, Rastad J, Ekbom A: Clinical presentation of primary hyperparathyroidism in Europe: nationwide cohort analysis on mortality from nonmalignant causes. J Bone Miner Res 2002, 17(Suppl 2): N68-N74.

7. Øgard CG, Engholm G, Almdal TP, Vestergaard H: Increased mortality in patients hospitalized with primary hyperparathyroidism during the period 1977-1993 in Denmark. World J Surg 2004, 28:108-111.

8. Wermers RA, Khosla S, Atkinson EJ, Grant CS, Hodgson SF, O'Fallon WM, et al: Survival after the diagnosis of hyperparathyroidism: a populationbased study. Am J Med 1998, 104:115-122.

9. Silverberg SJ, Shane E, Jacobs TP, Siris E, Bilezikian JPA: 10-year prospective study of primary hyperparathyroidism with or without parathyroid surgery. N Engl J Med 1999, 341:1249-1255.

10. Chambless LE, Folsom AR, Clegg LX, Sharrett AR, Shahar E, Nieto FJ, et al: Carotid wall thickness is predictive of incident clinical stroke: the Atherosclerosis Risk in Communities (ARIC) study. Am J Epidemio/ 2000, 151:478-487.

11. O'Leary DH, Polak JF, Kronmal RA, Manolio TA, Burke GL, WolfsonJr SK: Carotid-artery intima and media thickness as a risk factor for myocardial infarction and stroke in older adults. Cardiovascular Health Study Collaborative Research Group. N Engl J Med 1999, 340:14-22.

12. Burke GL, Evans GW, Riley WA, Sharrett AR, Howard G, Barnes RW, et al: Arterial wall thickness is associated with prevalent cardiovascular disease in middle-aged adults. The Atherosclerosis Risk in Communities (ARIC) Study. Stroke 1995, 26:386-391.

13. Nuzzo V, Tauchmanovà L, Fonderico F, Trotta R, Fittipaldi MR, Fontana D, et al: Increased intima-media thickness of the carotid artery wall, normal blood pressure profile and normal left ventricular mass in subjects with primary hyperparathyroidism. Eur J Endocrinol 2002, 147:453-459.

14. Rubin MR, Rundek T, McMahon DJ, Lee HS, Sacco RL, Silverberg SJ: Carotid artery plaque thickness is associated with increased serum calcium levels: the Northern Manhattan study. Atherosclerosis 2007, 194:426-432.
15. Foley Robert N, Collins Allan J, Areeflshani, Kalra Philip A: Calciumphosphate levels and cardiovascular disease in community-dwelling adults: The Atherosclerosis Risk in Communities (ARIC) Study. Am Heart J 2008, 156(3):556-563.

16. Cifuentes M, Fuentes C, Mattar P, Tobar N, Hugo E, Ben-Jonathan N, et al: Obesity-associated proinflammatory cytokines increase calciumsensing receptor (CaSR) protein expression in primary human adipocytes and LS14 human adipose cell line. Arch Biochem Biophys 2010, 500:151-156.

17. Pujia A, Gnasso A, Irace C, Colonna A, Mattioli PL: Common carotid arterial wall thickness in NIDDM subjects. Diabetes Care 1994, 17:1330-6.

18. White AD, Folsom AR, Chambless LE, et al: Community surveillance of coronary heart disease in the Atherosclerosis Risk in Communities (ARIC) Study: methods and initial two years' experience. J ClinEpidemiol 1996, 49:223-233

19. Walker MD, Fleischer J, Rundek T, McMahon DJ, Homma S, Sacco R, et al: Carotid Vascular Abnormalities in Primary Hyperparathyroidism. J ClinEndocrinolMetab 2009, 94:3849-3856.

20. Bots ML, Iglesias del Sol A, Grobbee DE: Carotid intima-media thickness measurements in observational and intervention studies. CurrResVasc Dis 1998, 3:274-283.

21. Fujii K, Abe I, Ohya Y, Onaka U, Tominaga M, Ohmori S, et al: Association between hyperinsulinemia and intima-media thickness of the carotid artery in normotensive men. J Hypertens 1997, 15:167-172

22. Kim MK, Kim G, Jang EH, Kwon HS, Baek KH, Oh KW, et al: Altered calcium homeostasis is correlated with the presence of metabolic syndromeand diabetes in middle-aged and elderly Korean subjects: the Chungju MetabolicDisease Cohort study (CMC study). Atherosclerosis 2010, 212:674-81.

23. Yonghan He, Huaqi Zhang JianghuaTeng, Lina Huang, Ying Li, Changhao Sun: Involvement of calcium-sensing receptor in inhibition of lipolysis throughintracellular cAMP and calcium pathways in human adipocytes. Biochem Biophys Res Commun 2011, 404:393-399. doi:10.1016/j. bbrc.2010.11.129.

24. Chow JY, Estrema C, Orneles T, Dong X, Barrett KE, Dong H: Calciumsensing receptor modulates extracellular $\mathrm{Ca}(2+)$ entry via TRPC-encoded receptor-operated channels in human aortic smooth muscle cells. Am J Physiol Cell Physiol. 2011, 301:C461-8.

25. Kim JY, Kim N, Yenari MA, Chang W: Mild Hypothermia Suppresses Calcium-Sensing Receptor (CaSR) Induction Following Forebrain Ischemia While Increasing GABA-B Receptor 1 (GABA-B-R1) Expression. Trans/ Stroke Res 2011, 1;2:195-201.

26. Brix JM, Stingl H, Höllerl F, Schernthaner GH, Kopp HP, Schernthaner G: Elevated Fetuin-A concentrations in morbid obesity decrease after dramatic weight loss. J ClinEndocrino/Metab. 2010, 95:4877-81.

27. Mori K, Emoto M, Inaba M: Fetuin-A and the cardiovascular system. AdvClin Chem 2012, 56:175-95.

doi:10.1186/1479-5876-10-114

Cite this article as: Montalcini et al:: Serum calcium level is related to both intima-media thickness and carotid atherosclerosis: a neglect risk factor in obese/overweight subjects. Journal of Translational Medicine 2012 10:114

\section{Submit your next manuscript to BioMed Central and take full advantage of:}

- Convenient online submission

- Thorough peer review

- No space constraints or color figure charges

- Immediate publication on acceptance

- Inclusion in PubMed, CAS, Scopus and Google Scholar

- Research which is freely available for redistribution 